\title{
Editorial: Recent Trends in Computational Fluid Dynamics
}

\author{
M. M. Bhatti ${ }^{1 \star}$, M. Marin ${ }^{2}$, A. Zeeshan ${ }^{3}$ and Sara I. Abdelsalam ${ }^{4}$ \\ ${ }^{1}$ College of Mathematics and Systems Science, Shandong University of Science and Technology, Qingdao, China, ${ }^{2}$ Department \\ of Mathematics and Computer Science, Transilvania University of Brasov, Brasov, Romania, ${ }^{3}$ Department of Mathematics and \\ Statistics, FBAS, IIU, Islamabad, Pakistan, ${ }^{4}$ Basic Science, Faculty of Engineering, The British University in Egypt, Al-Shorouk \\ City, Egypt
}

Keywords: computational fluid dynamics, non-Newtonian/Newtonian fluids, heat and mass transfer, multiphase flow simulations, thermodynamics, nanofluids

\section{Editorial on the Research Topic}

\section{Recent Trends in Computational Fluid Dynamics}

Computational fluid dynamics (CFD) [1] can be described as the set of techniques that assist the computer to provide the numerical simulation of the fluid flows. The three basic principles that can determine the physical aspects of any fluid are the i) energy conservation, ii) Newton's second law, and the iii) mass conservation. These flow problem can be described in terms of these basic laws. Mathematical equations, which are usually in the form of partial differential equations, portrayed the fluid behavior in the flow domain.

The solutions and interactive behavior of solid boundaries with fluid or interaction between the layers of the fluid while flowing are visualized using some CFD techniques. CFD helps replace these differential equations of fluid flow into numbers, and these numbers are beneficial in time and/or space which enable a numerical picture of the complete fluid flow. CFD is powerful in examining a system's behavior, beneficial, and more innovative in designing a system [2]. Also, It is efficient in exploring the system's performance metrics, whether it is for the yielding higher profit margins or in enhancing operational safety, and in various advantageous features [3].

Nowadays, CFD techniques are usually applied in various fields [4-8] i.e. car design, turbomachinery, ship design, and aircraft manufacturing. Moreover, it is beneficial in astrophysics, biology, oceanography, oil recovery, architecture, and meteorology. Numerous numerical Algorithm and software have been developed to perform CFD analysis. Due to the recent advancement in computer technology, numerical simulation for physically and geometrically complex systems can also be evaluated using PC clusters. Large scale simulations in different fluid flow on grids containing millions and trillions of elements can be achieved within a few hours via supercomputers. However, it is completely incorrect to think that CFD describes a mature technology, there are numerous open questions related to heat transfer, combustion modeling, turbulence, and efficient solution methods or discretization methods, etc. The coupling between CFD and other disciplines required further research, therefore, the main goal of this issue is to fill an essential gap that is greatly missed in this field. We sincerely hope that this issue will be beneficial to the readers to present the recent findings in the field and shed some light on the industrial sector.

Rafique et al. [9] used Buongiorno model to discuss the Casson nanofluid boundary layer flow through an inclined surface under the impact of Dufour and Soret. This nonlinear model is beneficial to understand the mechanism of heat and mass transfer by contemplating various essential features of the proposed boundary layer. Further, the Keller-box technique has been used to simulate the results. The results show that the Dufour effect has a strong impact on the temperature profile and 
that the thermophoresis produces an inverse impact on the concentration profile as compared with the temperature profile.

Shah et al. [10] investigated the CVFEM simulation to determine the nanoparticle's migration toward a permeable domain. The considered fluid model contains aluminum oxide nanoparticles. Darcy law, thermal radiation, Lorentz force, and shape factor. The proposed approach is beneficial for the two common schemes of CFD. In the proposed study, it was found that higher convection occurs due to the great influence of shape factor. According to the authors' simulation, it was shown that the magnetic field and temperature gradient have an inverse relationship. Later, Shah et al. [11] studied the behavior of couple stress fluid and nonisothermal convection with magnetic effects over a nonlinear sheet. Analytical simulation with the help of homotopy analysis method has been proposed for the solutions. According to their study, they found that the primary velocity faces significant resistance during the flow. In their proposed simulation, they noticed that magnetic effects produce resistance in the angular velocity, but enhances the temperature profile. Also, the Grashof number and Hall effects show a positive response to the temperature profile.

Shah et al. [12] contemplated the Mohand decomposition scheme to examine the Kortewege-De Vries equations. The fractional derivatives are expressed by Caputo fractional derivative operator. The validation and effectiveness of this scheme have been determined using numerical examples for integer order and fractional problems. According to their results, they concluded that the proposed scheme is easily adaptable, straightforward, and beneficial to solve nonlinear problems.

Irfan et al. [13] investigated the magnetized nanofluid motion with variable features propagating through a radiatively stretching sheet. Their proposed scheme was a numerical shooting method and the bvp4c built-in command in MATLAB. It was noticed that the thermophoresis, thermal conductivity, radiation parameter, and Brownian motion boost the thermal boundary layer. Further, in the proposed simulation, it was found that the Prandtl number suppresses the thermal profile. On the other hand, Brownian motion and Lewis numbers were seen to cause a strong influence on concentration profile, whereas the thermophoretic force was seen to produce and opposite effects. Later, Irfan et al. [14] used computational formulation, i.e., simplified finite difference scheme to establish and discuss the effects of porosity, thermal radiation, a magnetic and electric field with heat generation and absorption. A comparative study is also given using the simplified finite difference scheme and bvp4c where it was noticed that the model has a higher convergence rate.

Shafiq et al. [15] examined and discussed the motion of carbon nanotubes (CNTs) (single- and multi-walled) over a Riga plate. The Riga plate is filled with water as a base fluid. They used the Marangoni model for the fully developed electromagnetohydrodynamics flow. They proposed homotopy analysis method for the graphical and numerical outcomes. They noticed that multi-walled CNTs have higher velocity as compared with single-walled CNTs. They found similar outcomes of the magnetic field on temperature as already done by Shah et al. [11].
Bilal et al. [16] used a similar scheme used by Rafique et al. [9] to examine flow behavior betwixt a pair of rotating disks. They used the theory of the Cattaneo-Christov and Darcy model to formulate the proposed formulation. Further, Karman transformations have been used to model the mathematical modeling and numerical outcomes presented using the finite difference approach. They found that a higher Reynolds number produces resistance in the radial and axial velocities at the lower disk as compared with the upper disk. Further, the thermal profile was reduced due to the strong impact of the Prandtl number. At the lower disk, the shear drag coefficient diminishes while at the upper disk, the wall shear coefficient increases. Later, Ullah et al. [17] considered a similar geometry [16] with a three-dimensional Darcy-Forchheimer model and nanofluid flow. A computational shooting scheme was used to operate the proposed formulation. They found that the Darcy-Forchheimer model effects are negligible on the concentration and temperature profile.

Ahmed et al. [18] analyzed the concealed behavior of thermally radiative and magnetically influenced $\gamma \mathrm{Al}_{2} \mathrm{O}_{3}-\mathrm{H}_{2} \mathrm{O}$ and $\mathrm{Al}_{2} \mathrm{O}_{3}-\mathrm{H}_{2} \mathrm{O}$ nanofluid flow through a wedge. Combined simulation of shooting and RK scheme was used to evaluate the numerical outcomes. Their simulation shows that the Hartree pressure gradient significantly enhances the nanofluids velocity. The proposed composition of $\gamma \mathrm{Al}_{2} \mathrm{O}_{3}-\mathrm{H}_{2} \mathrm{O}$ and $\mathrm{Al}_{2} \mathrm{O}_{3}-\mathrm{H}_{2} \mathrm{O}$ becomes denser due to the strong impact of volume fraction and accordingly opposes the velocity field. The thermal profile $\gamma \mathrm{Al}_{2} \mathrm{O}_{3}-\mathrm{H}_{2} \mathrm{O}$ and $\mathrm{Al}_{2} \mathrm{O}_{3}-\mathrm{H}_{2} \mathrm{O}$ rises for higher volume fraction.

Ahmed and Khan [19] examined the mechanism of sodiumalginate $\left(\mathrm{C}_{6} \mathrm{H}_{9} \mathrm{NaO}_{7}\right)$ through a vertical heated plate with acceleration. Further, they contemplated the effects of convection and discussed the entropy generation. Laplace transforms with a combination of integral transforms that were used to generate the exact results. It was concluded that the maximal entropy can be achieved by taking higher values of Brinkmann number, fluid parameter, and Grashof number. It was also noticed that the Bejan number can also be maximal if the Prandtl number is high. The proposed fluid model reveals a dual impact.

Bhatti et al. [20] performed a theoretical analysis of the blood flow under the suspension of nanoparticles and microorganisms through an anisotropic artery in a sinusoidal form. The authors investigated a nonlinear Sutterby fluid model as blood to examine the rheological effects. A perturbation approach was used to elaborate on the series solutions. In their analysis, it was found that the non-Newtonian effects are in favor to resist the flow. Further, they noticed that the wall shear stress diminishes due to the stenosis, nanoparticle, and thermal Grashof number. Moreover, The Peclet number was found to create resistance in the microorganism profile. The results of this study play a significant role in biomedical engineering. Riaz et al. [21] presented a study that is beneficial for the urinary tract infections when the flow is sinusoidal. This analysis is essential to examine white particles occurring in the urine. They investigated the flow in a curved configuration with flexible walls and filled with particles in a fluid. A lubrication theory and perturbation approach was used to formulate the governing equations. Further, they also carried out the numerical results for the pressure along the whole channel. 
Alzahrani et al. [22] investigated the magnetohydrodynamics of a $3 \mathrm{D}$ flow through a rotating permeable conduit under the effect of Dufour and Soret and viscous dissipation. A viscous electrically-conducting fluid is considered upon which applied a magnetic field. Suitable transformations are used to transform from a nonlinear partial differential system of equations to an ordinary system of equations after which results were computed numerically using the shooting method. Then the pertinent parameters affecting the physical variables of the flow field have been thoroughly investigated.

Sanni et al. [23] studied the MHD flow of an incompressible Maxwell fluid flow induced by a quadratic stretching sheet through a $2 \mathrm{D}$ boundary layer. A variable magnetic field was applied to the flow with heat transfer, thermal radiation, and viscous dissipation. The system of partial differential equations has been transformed into ordinary differential equations (ODEs) by using some similarity variables. Numerical results have been achieved to find solutions to the energy and momentum equations in a closed-form.

Ahmed et al. [24] studied the peristaltic micropolar fluid flow influenced upon by heat and mass transfer with the magnetic field. The system of governing equations has been presented using a curvilinear coordinate system where they were further reduced using a lubrication approximation. Solutions were then derived by implementing the finite difference method.

Khan et al. [25] explored the thermal Eyring-Powell nanoliquid with triple diffusion via a periodic-moving system. A combination of some important parameters, such as the porosity parameter and magnetic effect, was also discussed.

\section{REFERENCES}

1. Anderson JD, Wendt J. Computational fluid dynamics. New York, NY: McGraw-Hill (1995).

2. Andersson B, Andersson R, Håkansson L, Mortensen M, Sudiyo R, Van Wachem B. Computational fluid dynamics for engineers. Cambridge, England:Cambridge University Press (2011).

3. Ferziger JH, Perić M, Street RL. Computational methods for fluid dynamics. Berlin: Springer (2002).

4. Chen Q. Computational fluid dynamics of HVAC: successes and failures. Atlanta, GA: American Society of Heating, Refrigerating and Air-Conditioning Engineers, Inc. (1997).

5. Kamyar A., Saidur R., Hasanuzzaman M. Application of computational fluid dynamics (CFD) for nanofluids. Int J Heat Mass Transf (2012) 55(15-16): 4104-4115. doi:10.1016/j.ijheatmasstransfer.2012.03.052

6. Zakaria MS, Ismail F, Tamagawa M, Azi AF, Wiriadidjaya S, Basri AA, et al. Computational fluid dynamics study of blood flow in aorta using OpenFOAM. I Adv Res Fluid Mech Thermal Sci (2018) 43:81-9.

7. Kozelkov AS, Kurulin VV, Lashkin SV, Shagaliev RM, Yalozo AV. Investigation of supercomputer capabilities for the scalable numerical simulation of computational fluid dynamics problems in industrial applications. Comput Math Math Phys (2016) 56(8):1506-1516. doi:10.1134/s0965542516080091

8. Mader CA, Kenway GK, Yildirim A, Martins JR. ADflow: an open-source computational fluid dynamics solver for aerodynamic and multidisciplinary optimization. J Aero Inf Syst (2020) 17:1-20. doi:10.2514/1.i010796

9. Rafique K, Anwar MI, Misiran M, Khan I, Alharbi SO, Thounthong P, et al. Numerical solution of Casson nanofluid flow over a non-linear inclined surface with Soret and Dufour effects by Keller-Box method. Front Phys (2020) 7:1-21.

10. Shah Z, Babazadeh H, Kumam P, Shafee A, Thounthong P. Numerical simulation of magnetohydrodynamic nanofluids under the influence of
The Buongiorno's nanofluid theory was investigated through the thermophoretic and Brownian motion effects. Further, the homotopy algorithm was used in order to analyze the fluid flow in a non-dimensional form.

Karuppusamy et al. [26] examined an entropy generation of a nanofluid of third-order with slip effect. The flow investigated was caused by a stretchable sheet through a porous plate under the influence of thermal radiation. Several other influential effects were taken into accounts such as the non-Fourier heat flux, convective surface boundary, and nanoparticle concentration on zero mass flux conditions. Similarity variables have been used in order to solve the governing physical system of equations and modify it into a nonlinear system of ODEs. Results were obtained using the usual homotopy algorithm to discuss the outcomes of the analysis.

\section{AUTHOR CONTRIBUTIONS}

MMB and MM drafted the first version of the editorial. AZ and SIA revised the first draft and made contributions about papers they edited.

\section{FUNDING}

MMB was supported by the Cultivation Project of Young and Innovative Talents in Universities of Shandong Province (Nonlinear Sciences Research Team).

shape factor and thermal transport in a porous media using CVFEM. Fron Phys (2019) 7:2396. doi:10.3389/fphy.2019.00164

11. Shah Z, Kumam P, Dawar A, Alzahrani EO, Thounthong P. Study of the couple stress convective micropolar fluid flow in a hall MHD generator system. Front Phys (2020) 7:171. doi:10.3389/fphy. 2019 Nov 5.

12. Shah R, Farooq U, Khan H, Baleanu D, Kumam P, Arif M. Fractional view analysis of third order Kortewege-De Vries equations, using a new analytical technique. Front Phys (2020) 7:244. doi:10.3389/fphy.2019.00244

13. Irfan M, Farooq MA, Iqra T. Magnetohydrodynamic free stream and heat transfer of nanofluid flow over an exponentially radiating stretching sheet with variable fluid properties. Front Phys (2019) 7:186. doi:10.3389/fphy.2019. 00186

14. Irfan M, Farooq MA, Iqra T. A new computational technique design for EMHD nanofluid flow over a variable thickness surface with variable liquid characteristics. Front Phys (2020) 8:66. doi:10.3389/fphy.2020.00066

15. Shafiq A, Zari I, Khan I, Khan TS. Marangoni driven boundary layer flow of carbon nanotubes towards a Riga plate. Front Phys (2019) 7:215. doi:10.3390/ math7111087

16. Bilal S, Tassaddiq A, Majeed AH, Ali F, Malik MY. Computational and physical examination about the aspects of fluid flow between two coaxially rotated disks by capitalizing non-Fourier heat flux theory: finite difference approach. Front Phys (2020) 7:209. doi:10.3389/fphy.2019.00209

17. Ullah MZ, Serra-Capizzano S, Baleanu D. A numerical simulation for Darcy-Forchheimer flow of nanofluid by a rotating disk with partial slip effects. Front Phys (2020) 7:741-52. doi:10.3389/fphy.2019.00219

18. Naveed Ahmed A, Khan U, Mohyud-Din S-T, Khan I, Murtaza R, Hussain I, et al. Novel investigation and hidden effects of MHD and thermal radiations in viscous dissipative nanofluid flow models. Front Phys (2020) 8:75. doi:10.3389/ fphy.2020.00075

19. Ahmed TN, Khan I. Entropy generation in $\mathrm{C}_{(6)} \mathrm{H}_{(9)} \mathrm{NAO}_{(7)}$ fluid over an accelerated heated plate. Front Phys. (2020) 7:250. doi:10.3389/fphy.2019.00250 
20. Bhatti MM, Marin M, Zeeshan A, Ellahi R, Abdelsalam SI. Swimming of motile gyrotactic microorganisms and nanoparticles in blood flow through anisotropically tapered arteries. Front Phys (2020) 8:95. doi:10.3389/fphy.2020.00095

21. Riaz A, Sadiq MA. Particle-fluid suspension of a non-Newtonian fluid through a curved passage: an application of urinary tract infections. Front Phys (2020) 8:109. doi:10.3389/fphy.2020.00109

22. Alzahrani AK, Ullah MZ, Muhammad T. Numerical treatment for 3D squeezed flow in a rotating channel with Soret and Dufour effects. Front Phys (2020) 8:201. doi:10.3389/fphy.2020.00201

23. Sanni KM, Hussain Q, Asghar S. Heat transfer analysis for non-linear boundary driven flow over a curved stretching sheet with a variable magnetic field. Front Phys (2020) 8:113. doi:10.3389/fphy.2020.00113

24. Ahmed R, Ali N, Khan SU, Rashad AM, Nabwey HA, Tlili I. Novel microstructural features on heat and mass transfer in peristaltic flow through a curved channel. Front Phys (2020) 8:178. doi:10.3389/fphy.2020.00178

25. Khan SU, Vaidya H, Tlili I, Prasad KV. Triple diffusive unsteady flow of Eyring-Powell nanofluid over a periodically accelerated surface with variable thermal features. Front Phys (2020) 8:246. doi:10.3389/fphy 2020.00246

26. Karuppusamy L, Muhiuddin G, Alanazi AM, Alkhaldi AH, Rajan S. Entropy optimization of 3rd-grade nanofluid slip flow embedded in a porous sheet with zero mass flux and non-Fourier heat flux model. Front Phys (2020) 8:250.

Conflict of Interest: The authors declare that the research was conducted in the absence of any commercial or financial relationships that could be construed as a potential conflict of interest.

Copyright (c) 2020 Bhatti, Marin, Zeeshan and Abdelsalam. This is an open-access article distributed under the terms of the Creative Commons Attribution License (CC $B Y)$. The use, distribution or reproduction in other forums is permitted, provided the original author(s) and the copyright owner(s) are credited and that the original publication in this journal is cited, in accordance with accepted academic practice. No use, distribution or reproduction is permitted which does not comply with these terms. 